\title{
Los balances hídricos agrícolas en modelos de simulación agroclimáticos. Una revisión analítica
}

\section{Agricultural water balances in agroclimatic simulation models. An analytical review}

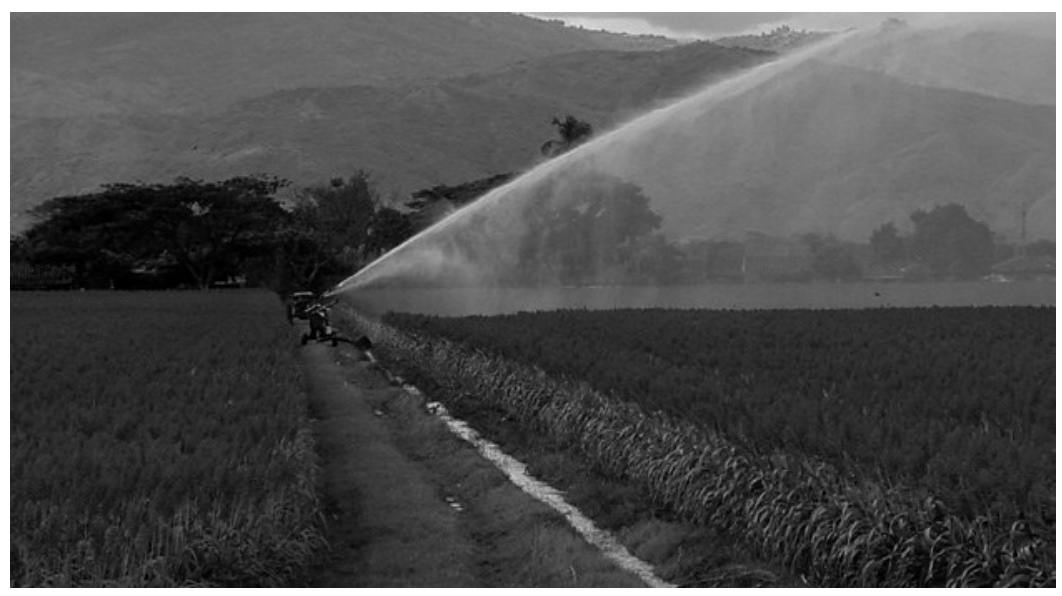

JOSÉ ALEJANDRO CLEVES L., 3

JAVIER TORO C. ${ }^{2}$

LIVEN FERNANDO MARTÍNEZ B. ${ }^{2}$

\section{RESUMEN}

En este artículo se analiza la aplicabilidad de los modelos agroclimáticos de simulación de cultivos CropWat v 8.0 y AquaCrop bajo la ocurrencia de los eventos ENOS (E1 Niño-Oscilación del Sur) y de cambio climático. Igualmente se resalta la importancia de estos modelos porque permiten a los agricultores implementar medidas de manejo y ubicación de cultivares teniendo en cuenta las condiciones climáticas para participar desde el contexto territorial en el diseño e implementación de estrategias de mitigación y/o adaptación. De manera complementaria se comparan las diferentes metodologías utilizadas para la determinación de la evapotranspiración de referencia $\left(\mathrm{ET}_{\mathrm{o}}\right)$ y se describen los atributos, características y aplicabilidad en la ecuación Penman-Monteith. Finalmente se define las variables del balance hídrico agrícola, indicando su aplicabilidad en la conservación o aumento de la productividad en diferentes escenarios. Se concluye que estos modelos son herramientas técnicas fundamentales en la planificación de siembras y de cosechas.

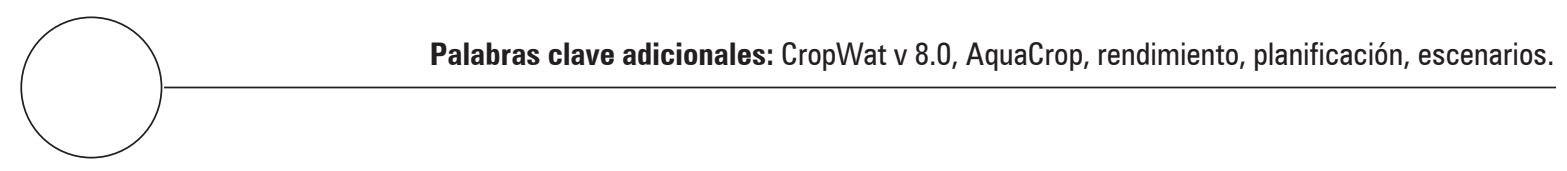

\footnotetext{
Facultad Seccional Duitama, Escuela Administración de Empresas Agropecuarias, Universidad Pedagógica y Tecnológica de Colombia (UPTC), Duitama (Colombia).

2 Instituto de Estudios Ambientales (IDEA), Universidad Nacional de Colombia, Bogotá (Colombia).

3 Autor para correspondencia: jaclevesl@uptc.edu.co
} 


\section{ABSTRACT}

This article analyzes the applicability of the agroclimatic crop simulation models CropWat $v 8.0$ and AquaCrop in the occurrence of ENSO (El Niño-Southern oscillation) events and climate change. The importance of these models is significant because they help farmers implement management measures and locate cultivars taking into account climatic conditions, thus allowing their participation, from the territorial context, in the design and implementation of strategies for mitigation and/or adaptation. Different methodologies used for determining the reference evapotranspiration $\left(\mathrm{ET}_{\mathrm{o}}\right.$ ) were compared, and the attributes, characteristics and applicability with the Penman-Monteith equation were described. Finally, the variables of the agricultural water balance were determined, along with their applicability regarding the preservation or the increase of productivity in different scenarios. It was concluded that these models are essential technical tools for planning sowing and harvests.

Additional keywords: CropWat v 8.0, AquaCrop, performance, planning, scenarios.

Fecha de recepción: 05-01-2016

Aprobado para publicación: 14-05-2016
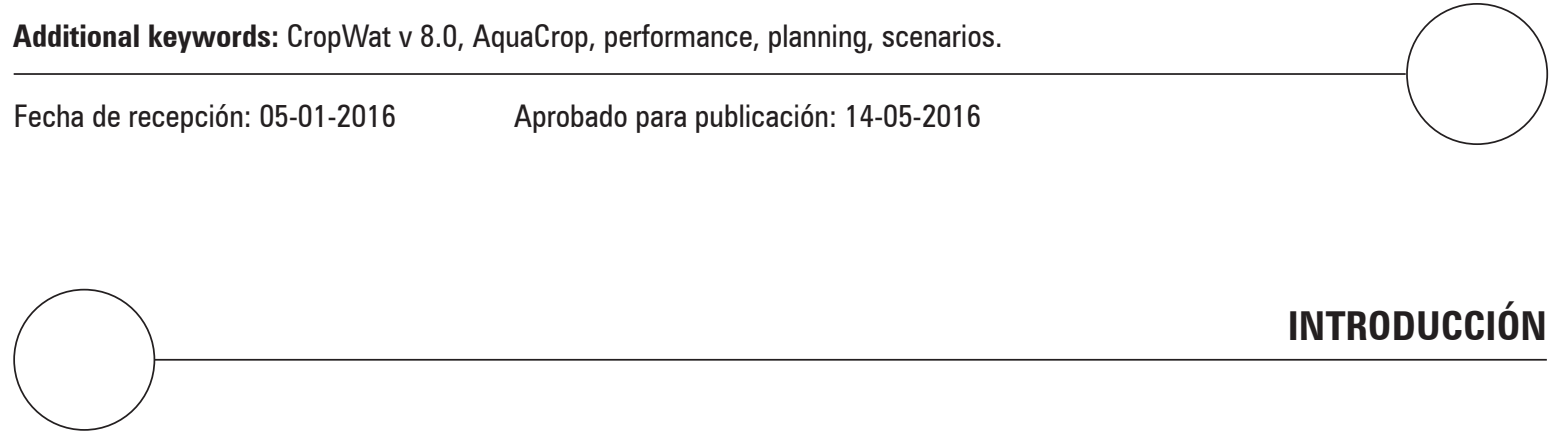

INTRODUCCIÓN

La intensidad de las oscilaciones de la temperatura superficial (TSM) del océano Pacífico en el sector 3.4 asociada a los fenómenos ENOS 4 , tiene una marcada incidencia en la variabilidad climática interanual del territorio colombiano (Córdoba et al., 2015; Espinoza et al., 2009; Lagos et al., 2008; Palacios et al., 2009). El conocimiento de esta anomalía y el uso de herramientas como los modelos de simulación, aportan información relevante para aumentar la resiliencia cultural de los agroecosistemas.

Los modelos de simulación CropWat v8.0 y AquaCrop estiman el requerimiento hídrico de los cultivos (Bouraima et al., 2015; Charchousi et al., 2014; Dechmi et al., 2003; Silva et al., 2015), al igual que su rendimiento o el porcentaje de reducción según las condiciones climáticas o meteorológicas en un lugar y momento determinado (Andarzian et al., 2011; Araya et al., 2010; García-Vila y Fereres, 2012; Vanuytrecht et al., 2014). Son útiles para definir las fechas óptimas de siembra en relación con las predicciones climáticas en condiciones de años análogos bajo la influencia de anomalías climáticas, asociadas al fenómeno El Niño-Oscilación del Sur (ENSO o ENOS). Para su aplicación es necesario analizar la información de la fenología, características del cultivo, propiedades hidrofísicas del suelo y manejo agronómico.

4 El ENOS es un fenómeno dinámico oceánico-atmosférico que consiste en la interacción de las aguas superficiales del océano Pacífico tropical con la atmósfera circundante. El ENOS está relacionado con alteraciones de las principales variables climatológicas en muchas regiones del mundo, incidiendo significativamente en ecosistemas terrestres y marinos. Tiene dos componentes, un componente oceánico en el cual se diferencian la fase cálida y la fase fría (El Niño, La Niña); y un componente atmosférico cuantificado como la diferencia estandarizada de presiones atmosféricas entre los sectores occidental (Darwin, Australia) y el sector oriental-central del Océano Pacífico Tropical (alrededores de la isla Tahití), denominado Índice de Oscilación del Sur (IOS o SOI) (Solano y Stolz, 2015; Trenberth, 1976; Yuan y Di, 2014). 
En Colombia los modelos CropWat v8.0 y AquaCrop son los de mayor uso, debido a la difusión hecha por la Organización de la Naciones Unidas para la Alimentación y la Agricultura (FAO), la academia, los gremios y los productores. Adicionalmente requieren un número reducido de parámetros edáficos, información del cultivo y de variables climáticas en diferentes escalas (diaria, décadal o mensual) y para su ejecución hay disponible un programa de computación o software de acceso libre diseñado en versión para Windows ${ }^{\circledR}$ llamado CROPWAT v 8.0, que puede ser consultado y/o descargado de la página web: http://www.fao.org/nr/water/infores_databases_cropwat.html. Por estas razones son reconocidos mundialmente como modelos robustos y de fácil manejo (Bernal et al., 2012; García et al., 2015).

Los modelo de simulación son herramientas que facilitan la toma de decisiones en los procesos de evaluación de tierras para fines agrícolas, porque estiman el requerimiento hídrico de los cultivos y el rendimiento y su porcentaje de reducción, según las condiciones climáticas o meteorológicas de un lugar en particular (Bernal et al., 2013a; Bernal et al., 2013b; Cortés et al., 2013; Méndez et al., 2013; Bernal et al., 2012).

\section{BALANCE HÍDRICO}

El balance hídrico se realiza utilizando datos reales o simulados de precipitación e información climatológica, con el fin de calcular el requerimiento hídrico de una zona en particular (Claro, 1991; Santillán et al., 2013; De Souza et al., 2006). Hay diferentes tipos de balances hídricos: climáticos, agroclimáticos, hidrológicos, agroforestales, de cuencas, de drenaje y riego. En el presente documento se hará alusión exclusivamente a los balances hídricos agrícolas.

\section{Componentes generales del balance hídrico agrícola}

En el caso de un suelo con vegetación, el balance hídrico simplificado se calcula como se presenta en la ecuación 1:

$$
P=E T+\Delta H S+I+E
$$

Donde:

$P=$ Precipitación (más riego, si se suministra).

ET $=$ Evapotranspiración.

$\Delta H S=$ Cambios de la humedad del suelo: (humedad final-humedad inicial).

$I=$ Infiltración de las capas profundas.

$E=$ Escorrentía

\section{Precipitación: P}

En la mayoría de los casos, la precipitación representa la mayor parte de la totalidad del aporte hídrico al suelo (Claro, 1991). Del agua que se precipita sobre la superficie del terreno, parte es interceptada por la vegetación (intercepción foliar), otra parte se infiltra y se incorpora a la capa radical, un porcentaje percola debajo de las raíces del cultivo, y otra parte escurre sobre la superficie del terreno (Echeverría et al., 2007). La proporción de agua retenida en la capa radical, almacenada en esa zona para ser usada en evapotranspiración del sistema planta-suelo, depende de las características del terreno para captar agua (condiciones físicas y químicas del suelo, grado de humedad, cobertura, pendiente) y de las características de la lluvia (altura, intensidad, duración y frecuencia). Esta porción de la lluvia se denomina precipitación efectiva (Saseendran et al., 2015). 
La precipitación efectiva $\left(P_{e}\right)$ significa lluvia útil o utilizable, es parte de la precipitación total que satisface directamente las necesidades de agua de los cultivos (Dastane, 1978). La intensidad o la cantidad que se recibe de lluvia no es necesariamente útil o deseable en el momento; una parte puede ser usada mientras que otra puede generar efectos ambientales negativos. Por lo anterior, en los cálculos debe tenerse en cuenta la precipitación efectiva $\left(\mathrm{P}_{\mathrm{e}}\right)$, que es igual a la precipitación (P) multiplicada por un coeficiente menor que la unidad. Con base en los datos de velocidad de infiltración y de intensidad de precipitación (ambas variables en función de tiempo) es posible calcular la $\left(\mathrm{P}_{\mathrm{e}}\right.$; equación 2$)$ para un determinado evento, igualmente puede llevarse a cabo utilizando la ecuación propuesta por el Departamento de Agricultura de Estados Unidos (USDA) (FAO, 1992).

$P_{e}=\left(1,25247 * \mathrm{P}_{t}^{0,82416}-2,93522\right) * 10^{0,00095 U} * f(2)$

Dónde:

$P_{e}=$ Precipitación efectiva mensual $(\mathrm{mm})$.

$P_{t}=$ Precipitación total mensual $(\mathrm{mm})$.

$U=$ Uso consuntivo medio mensual.

$f=0,531747+0,011621 * \Delta s-8.9 \times 10^{-5} * \Delta s^{2}+2,3$ $x 10^{-7} * \Delta s^{3}$

Donde:

$\Delta s=$ Dosis de riego neta $(\mathrm{mm})$.

En el contexto agrícola la lluvia se considera efectiva hasta donde satisface las necesidades hídricas para la preparación del terreno, consumo por el cultivo, requerimientos de filtrado salino, y/o necesidades de percolación (como en el arroz y estanques piscícolas).

\section{Evapotranspiración de referencia: ETo}

Corresponde a la evaporación de agua localizada en el suelo y la traspiración de agua de las plantas. La tasa de evapotranspiración de una superficie de referencia, que ocurre sin restricciones de agua, se conoce como evapotranspiración del cultivo de referencia (ETo) (Li et al., 2015; Westerhoff, 2015). La superficie de referencia corresponde a un cultivo hipotético de pasto en óptimas condiciones fitosanitarias y nutricionales, con una altura uniforme de $0,12 \mathrm{~m}$, una resistencia superficial fija de $70 \mathrm{~s} \mathrm{~m}^{-1}$ y un albedo de 0,23 , que crece activamente y da sombra totalmente al suelo. La resistencia superficial fija de $70 \mathrm{~s} \mathrm{~m}^{-1}$ implica un suelo adecuadamente regado, hasta llegar a capacidad de campo (FAO, 2006; Spano et al., 2009).

La evapotranspiración se expresa en milímetros $(\mathrm{mm})$ por unidad de tiempo. Esta unidad expresa la cantidad de agua perdida por una superficie cultivada en unidades de altura de agua. La unidad de tiempo puede ser 1 hora, 1 día, 10 días, 1 mes o incluso un completo período de cultivo o un año (FAO, 2006).

Esta variable es fundamental para el cálculo de la evapotranspiración de los cultivos y por lo tanto del balance hídrico, de donde se derivan variables de análisis como época y cantidad de déficit, excesos, almacenaje en el suelo, cambio en el almacenaje y pérdida por escorrentía, entre otras.

\section{Coeficiente del cultivo: Kc}

Los efectos combinados de la transpiración del cultivo y la evaporación del suelo se integran en un coeficiente único del cultivo. El coeficiente único del cultivo $(\mathrm{Kc})$ incorpora las características del cultivo y los efectos promedios de la evaporación en el suelo (FAO, 2006), tal como se indica en la ecuación 3. 


$$
K c=E T c / E T o
$$

Donde:

$E T_{C}=$ Evapotranspiración real del cultivo.

$E T_{O}=$ evapotranspiración de referencia.

Algunos factores que afectan los valores de $\mathrm{K}_{c}$ son: (1) las diferencias en la morfología de la planta, que afecta la intercepción de luz y la resistencia aerodinámica, (2) la fisiología vegetal, incluidos los efectos sobre el número, la distribución y las respuestas de los estomas al ambiente, (3) el sistema de riego que afecta la distribución de la humedad del suelo, (4) la frecuencia del riego y las precipitaciones y (5) la interceptación de rocío y condensación (neblina) que afecta la resistencia del dosel (Spano et al., 2009).

\section{Evapotranspiración del cultivo: $\mathrm{ET}_{\mathrm{C}}$}

La evapotranspiración del cultivo $\left(E T_{c}\right)$ expresa la cantidad total de agua perdida por la superficie de un cultivo en forma de vapor. La evapotranspiración es la suma de la evaporación de la superficie del suelo y la transpiración de la planta $(\mathrm{FAO}$, 1977). Por su parte la $\mathrm{ET}_{\mathrm{c}}$ expresa la evapotranspiración de un cultivo que se desarrolla libre de enfermedades, con buena fertilización, que crece en un campo extenso bajo condiciones óptimas de humedad en el suelo y alcanza su producción total bajo ciertas condiciones climáticas (FAO, 2006). El cálculo de la $\mathrm{ET}_{\mathrm{c}}$ se representa en la ecuación 4 (Casa et al., 2000; Pereira et al., 2011).

$$
E T c=K c * E T o
$$

Donde:

$$
\begin{aligned}
& K_{C}=\text { Coeficiente del cultivo. } \\
& E T_{O}=\text { Evapotranspiración de referencia } .
\end{aligned}
$$

En la tabla 1 se presentan diferentes métodos utilizados para calcular la evapotranspiración, con sus principales atributos y en la tabla 2 pueden consultarse las principales ecuaciones.

\section{METODOLOGÍAS PARA ESTIMAR EL BALANCE HÍDRICO}

El balance hídrico permite integrar las variables del clima para determinar las necesidades de riego en un periodo determinado con el fin de planificar, diseñar u operar la disponibilidad del recurso hídrico en una zona determinada (Guarnizo, 2007). Pérez (1992) plantea que para la estimación del balance hídrico es indispensable el cálculo de la evapotranspiración que se realiza con diferentes metodologías: Holdridge, Budyko, Ivanov, Lorry y Jhonson, Papadakis, Thornthwaite, Linacre, Makkink, Turc, Harvgreaves, Christiansen y Penman.

Un método ampliamente utilizado para el cálculo de la evapotranspiración potencial (ETP) hasta la década de 1960 fue el método empírico de Thornthwaite, el cual fue desarrollado para estimar la $\mathrm{ET}_{\mathrm{p}}$, usando la temperatura del aire como el principal parámetro (Bruno et al., 2007).

Este método aunque fue ampliamente difundido, presentó inconvenientes conceptuales al no tener en cuenta restricciones como la disponibilidad del agua del suelo y al considerar que su magnitud dependía exclusivamente de las condiciones meteorológicas. En su cuantificación se definieron con poca precisión las características de la superficie evaporante (referencia), lo que condujo a errores al subestimar la evapotranspiración de referencia (real) de un cultivo.

Debido a que la evapotranspiración de algunos cultivos altos es superior a la $\mathrm{ET}_{\mathrm{p}}$ (por ejemplo en zonas áridas y semiáridas), se hizo necesario 
introducir el concepto de evapotranspiración del cultivo de referencia $\left(E T_{0}\right)$ mediante la ecuación de Penman Monteith, según la UNESCO como reportan Castillo et al. (2001).

El 26 de mayo de 2000, el panel de expertos de la FAO publicó el documento número 56 titulado "Evapotranspiración del cultivo: guías para la determinación de los requerimientos de agua de los cultivos" (Crop Evapotranspiration: guidelines for computing crop water requirements), donde se recomienda la adopción del método combinado de Penman-Monteith. Esta propuesta fue validada y aceptada por la comunidad científica mundial (Allen et al., 2006). Debido a la importancia histórica de esta ecuación se presenta a continuación.

Tabla 1. Comparación de los métodos para el cálculo de la evapotranspiración de referencia ETO (Jensen et al., 1990).

\begin{tabular}{|c|c|c|c|c|c|c|}
\hline \multirow{2}{*}{$\frac{\text { Tipo de clima }}{\text { Indicador }}$} & \multicolumn{3}{|c|}{ Húmedo } & \multicolumn{3}{|c|}{ Árido } \\
\hline & $\begin{array}{c}\text { Puesto } \\
\text { No. }\end{array}$ & Sobreestimación ${ }^{1}$ & $\begin{array}{c}\text { Error } \\
\text { estándar }{ }^{2}\end{array}$ & $\begin{array}{c}\text { Puesto } \\
\text { No. }\end{array}$ & Sobreestimación ${ }^{1}$ & Sobreestimación ${ }^{2}$ \\
\hline \multicolumn{7}{|c|}{ Métodos combinados } \\
\hline Penman-Monteith & 1 & $+4 \%$ & 0,32 & 1 & $-1 \%$ & 0,49 \\
\hline FA0-24 Penman $\mathrm{C}=1$ & 14 & $+29 \%$ & 0,93 & 6 & $+12 \%$ & 0,69 \\
\hline $\begin{array}{l}\text { FA0-24 Penman } \\
\text { (corregido) }\end{array}$ & 19 & $+35 \%$ & 1,14 & 10 & $+18 \%$ & 1,10 \\
\hline FA0-PPP-17 Penman & 4 & $+16 \%$ & 0,67 & 5 & $+6 \%$ & 0,68 \\
\hline Penman (1963) & 3 & $+14 \%$ & 0,60 & 7 & $-2 \%$ & 0,70 \\
\hline Penman 1963, VPD \#3 & 6 & $+20 \%$ & 0,69 & 4 & $+6 \%$ & 0,67 \\
\hline 1972 Kimberley Penman & 8 & $+18 \%$ & 0,71 & 8 & $+6 \%$ & 0,73 \\
\hline 1982 Kymberley Penman & 7 & $+10 \%$ & 0,69 & 2 & $+3 \%$ & 0,54 \\
\hline Businger-van Badel & 16 & $+32 \%$ & 1,03 & 11 & $+11 \%$ & 1,12 \\
\hline \multicolumn{7}{|c|}{ Métodos de radiación } \\
\hline Priestley Taylor & 5 & $-3 \%$ & 0,68 & 19 & $-27 \%$ & 1,89 \\
\hline FA0-Radiación & 11 & $+22 \%$ & 0,79 & 3 & $+6 \%$ & 0,62 \\
\hline \multicolumn{7}{|c|}{ Métodos de temperatura } \\
\hline Jensen-Haise & 12 & $-18 \%$ & 0,84 & 12 & $-12 \%$ & 1,13 \\
\hline Hargreaves & 10 & $+25 \%$ & 0,79 & 13 & $-9 \%$ & 1,17 \\
\hline Turc & 2 & $+5 \%$ & 0,56 & 18 & $-26 \%$ & 1,88 \\
\hline SCS Blaney-Criddle & 15 & $+17 \%$ & 1,01 & 15 & $-16 \%$ & 1,29 \\
\hline FA0 Blaney-Criddle & 9 & $+16 \%$ & 0,79 & 9 & $0 \%$ & 0,76 \\
\hline Thornwaite & 13 & $-4 \%$ & 0,86 & 20 & $-37 \%$ & 2,40 \\
\hline \multicolumn{7}{|c|}{ Métodos de tanque de evaporación } \\
\hline Tanque Clase A & 20 & $+14 \%$ & 1,29 & 17 & $+21 \%$ & 1,54 \\
\hline Christiansen & 18 & $-10 \%$ & 1,12 & 16 & $-6 \%$ & 1,41 \\
\hline FAO Clase A & 17 & $-5 \%$ & 1,09 & 14 & $+5 \%$ & 1,25 \\
\hline
\end{tabular}

${ }^{1}$ Sobre o subestimado como porcentaje de datos de 11 sitios con lisímetros, corregidos por el tipo de cultivo de referencia.

${ }^{2}$ Error estándar ponderado de las estimaciones mm día ${ }^{-1}$. 
Tabla 2. Principales ecuaciones para el cálculo de la evapotranspiración de referencia (ETo).

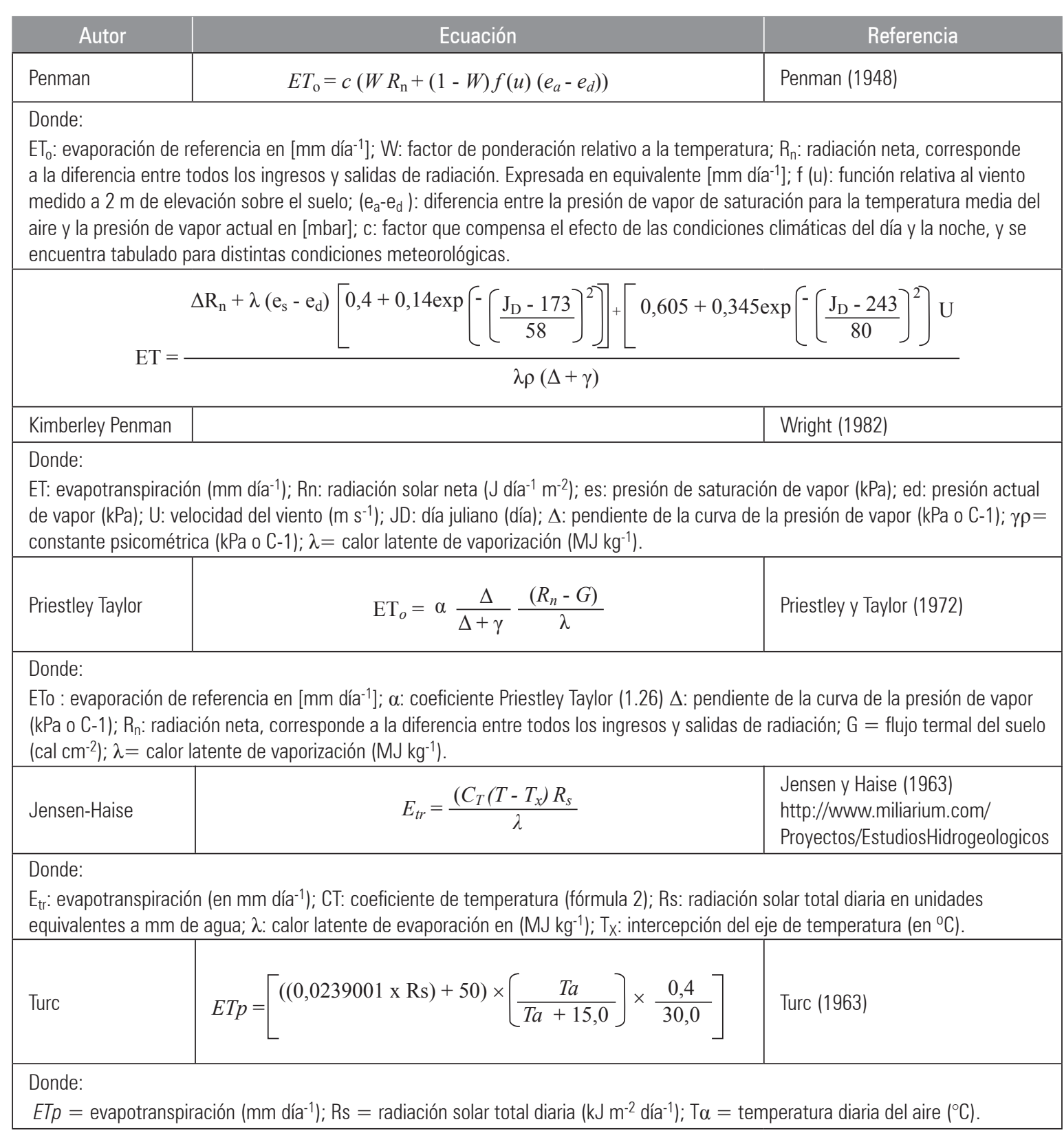

\section{ECUACIÓN DE PENMAN-MONTEITH (PME)}

La ecuación de Penman-Monteith ha sido ampliamente evaluada y aceptada por la comunidad científica nacional e internacional por presentar el menor error estándar y por ser la que más se ajusta a diferentes latitudes y climas, proporciona valores que son consistentes con datos reales de uso de agua de cultivos en diferentes latitudes (Bernal et al., 2013a). 
Esta ecuación superó las deficiencias de métodos recomendados anteriormente, incorpora parámetros fisiológicos y aerodinámicos, inclusive se han desarrollado procedimientos para la utilización, con pocos datos climáticos, básicamente se requiere disponer de información sobre las siguientes variables climatológicas (Ficklin et al., 2015): temperatura del aire, humedad relativa del aire o presión de vapor, velocidad del viento e insolación o radiación solar.

El empleo del método de Penman-Monteith proporciona resultados satisfactorios para calcular los efectos del clima sobre las necesidades de agua de los cultivos, al definir con exactitud un componente fundamental del balance hídrico denominado evapotranspiración del cultivo de referencia $\left(E T_{c}\right)$. Para la determinación de los requerimientos hídricos de cultivos en condiciones de $\mathrm{ET}_{\mathrm{c}}$, se debe tener en cuenta los coeficientes específicos del cultivo $\left(\mathrm{K}_{\mathrm{c}}\right)$, tal como se refirió anteriormente en la ecuación 4.

El enfoque del modelo Penman-Monteith incluye todos los parámetros de intercambio de energía y flujo de calor (evapotranspiración) de grandes extensiones uniformes de vegetación. La mayoría de los cuales son medidos o pueden calcularse fácilmente a partir de datos meteorológicos (FAO, 2006).

Por lo anterior el método FAO Penman-Monteith es el más adecuado para determinar la evapotranspiración de referencia $\mathrm{ET}_{\mathrm{O}}$. Se ha recomendado su uso por los equipos de investigación ASCE y europeo por generar resultados relativamente exactos y consistentes en climas áridos y húmedos.

La ecuación y definición de las variables de Penman-Monteith fueron extraídas del documento No. 56 de la FAO y se presenta en la ecuación 5 (Allen et al., 2006).

$$
\mathrm{ET}_{\mathrm{o}}=\frac{0,408 \Delta\left(\mathrm{R}_{\mathrm{n}}-\mathrm{G}\right)+\gamma \frac{900}{\mathrm{~T}+273} \mathrm{u}_{2}\left(\mathrm{e}_{\mathrm{s}}-\mathrm{e}_{\mathrm{a}}\right)}{\Delta+\gamma\left(1+0,34 \mathrm{u}_{2}\right)}
$$

Donde:

ETo = evapotranspiración del cultivo de

referencia $\left(m m\right.$ día $\left.^{-1}\right)$.

$\gamma^{*}=$ constante psicométrica modificada

utilizada en el método de Penman-Monteith

$(\mathrm{mbar} / \mathrm{C})$.

$e_{s}-e_{a}=$ déficit de presión de vapor $(\mathrm{mb})$.

$e_{s}=$ presión de vapor a saturación a la

temperatura promedio del aire $(\mathrm{mb})$.

$e_{a}=$ presión de vapor tomada a la temperatura

a punto de rocio (mb).

$L=$ calor latente de vaporización (cal/g).

$\Delta=$ pendiente de la curva de presión de

la saturación de vapor a una temperatura

especifica $\left(\right.$ mbar $\left./{ }^{\circ} \mathrm{C}\right)$.

$\gamma=$ constante psicométrica.

$R_{n}=$ energía de radiación neta $\left(\right.$ cal $\mathrm{cm}^{-2}$ dia $\left.^{-1}\right)$.

$T=$ temperatura promedio $\left({ }^{\circ} \mathrm{C}\right)$.

$G=$ flujo termal del suelo $\left(\mathrm{cal} \mathrm{cm}^{-2}\right)$.

$u_{2}=$ velocidad del viento a $2 \mathrm{~m}$ de altura. 
La evapotranspiración de referencia (ETo) provee un estándar de comparación mediante el cual se puede comparar la evapotranspiración en diversos períodos del año o en otras regiones y también se puede relacionar la evapotranspiración de diferentes cultivos.

\section{Aplicaciones de la ecuación de Penman-Monteith (PMe)}

Mediante esta propuesta se estandarizó el cálculo de la evapotranspiración de referencia (ETo) y se describieron los procedimientos para calcular los parámetros. El método está definido para un cultivo hipotético de referencia, similar a la evaporación presentada en una superficie extensa de pasto verde, sano, con una altura uniforme de $12 \mathrm{~cm}$, con crecimiento activo y con continua y oportuna disponibilidad de agua para riego (FAO, 2006; Liang et al., 2008).

El método permite su utilización con datos climáticos limitados, eliminando por lo tanto la necesidad de otros para la estimación de la evapotranspiración. De esta manera se crea una base robusta para la determinación universal de los cálculos de los requerimientos de agua de los cultivos. Las evaluaciones han demostrado que el método de Penman-Monteith es el que menos sobre o subestima la ETo; además, estadísticamente presenta menor error estándar en diferentes climas (tropical, árido y húmedo) (Jensen et al., 1990).

La ecuación tiene aplicaciones en los procesos de caracterización climática, al relacionarse la precipitación y la evaporación en escala mensual (Eslava, 1993). Es una herramienta útil en procesos de planeación del uso eficiente del suelo rural, por lo que puede ser incorporado en la formulación de los planes de ordenamiento territorial (POT).
El método Penman-Monteith ha sido incorporado posteriormente a los modelos ${ }^{5}$ agroclimáticos de simulación de cultivos CropWat v 8.0 y AquaCrop (Bouraima et al., 2015; Vanuytrecht et al., 2014) que calculan según las condiciones climáticas o meteorológicas del lugar los requerimientos hídricos de los cultivos, los rendimientos estimados y el porcentaje de reducción del rendimiento. Estos métodos se basan en las características del cultivo, propiedades hidrofísicas del suelo y en el manejo agronómico (Stedutto et al., 2009).

\section{MODELO CROPWAT V 8.0}

Es un programa de computación que puede ser usado para el cálculo de los requerimientos de agua de los cultivos y de sus requerimientos de riego con base en datos climáticos y de las características ecofisiológicas del cultivo (Stancalie et al., 2010). Está basado principalmente en la metodología presentada por la FAO en la serie de riego y drenaje, documento 24 (Doorenbos y Pruitt, 1977), el documento 33 (Doorenbos et al., 1979) y en la ecuación de Penman-Monteith, descrita por la FAO en el documento 56 (Allen et al., 2006).

Además, el programa permite la elaboración de calendarios de riego para diferentes condiciones de manejo y el cálculo del esquema de provisión de agua para diferentes patrones de cultivos (Stancalie et al., 2010), se ha usado en las diferentes condiciones edáficas y climáticas de las zonas productoras de papa, maíz, arroz, frijol, yuca y pastos (Boshell, 2013; Bernal et al., 2013a; Bernal et al., 2013b; Feng et al., 2007) y en la implementación de un sistema de alertas agroclimáticas tempranas (SAT) para apoyar la toma de decisiones de alternativas tecnológicas de manejo, tendientes a disminuir los efectos de eventos climáticos adversos (Corpoica, 2011; Pérez, 2013).

5 Los modelos son herramientas para sintetizar el conocimiento, probar hipótesis, describir y entender sistemas complejos y comparar diferentes situaciones, se usan como soporte para la toma de decisiones de manejo y planeación (Marcelis et al., 1998; López et al., 2011). 
Este modelo ayuda a definir criterios para el cálculo de la evapotranspiración de referencia (ETo) y del cultivo (ETc) con datos de variables del clima en versión mensual, decadal y diaria; analizar la lluvia efectiva; posibilita estimar los datos climáticos, en caso de no contar con los valores medidos; efectúa cálculos diarios y decadales de los requerimientos de agua del cultivo basados en algoritmos de cálculo actualizados, incluido el ajuste de los valores del coeficiente del cultivo; calcula las necesidades netas de agua de los cultivos; es útil para la programación de riego y para la elaboración de tablas de balances diarios de agua en el suelo; calcula el porcentaje de disminución del rendimiento del cultivo debido al estrés hídrico que eventualmente presentaría respecto a la predicción climática y finalmente estima la provisión de agua (Levine et al., 1998).

Igualmente el modelo se ha empleado para evaluar la demanda de agua de los cultivos, así como el diseño del plan de riegos mediante la simulación de la reducción del rendimiento potencial ${ }^{6}$ (RRP) del cultivo debido al estrés por el déficit de agua (Cavero et al., 2000; Mullick et al., 2011), también se ha usado en estudios regionales con Sistemas de Información Geográfica (SIG) sobre el balance de humedad del suelo, incluyendo información de imágenes de satélite en las áreas de riego en Irán (Jamshid, 2003) y para evaluar la uniformidad en la distribución del agua entre los usuarios al inicio y final de la aplicación de los módulos de riego en el distrito de riego 017, Comarca Lagunera, México (Santamaría et al., 2008), entre otras aplicaciones.

Para mayor información y consulta de ejemplos típicos de aplicación en el contexto colombiano del programa CropWat se puede consultar el siguiente vínculo: http://dapa.ciat.cgiar.org/ uso-de-cropwat-para-calcular-requerimientoshidricos-en-frijol-arbustivo.

\section{MODELO AQUACROP}

Es un modelo de gran funcionabilidad para el cálculo del rendimiento de cultivos herbáceos, desarrollado por la División de Tierra y Agua de la FAO, simula la respuesta en el rendimiento de acuerdo al agua transpirada por el cultivo, según el volumen de agua disponible en el suelo, estimado a partir de datos de lluvia y/o riego en el suelo. Permite estimar el balance hídrico/día/ mes/ciclo y la precipitación efectiva (Stedutto et al., 2009; Toumi et al., 2015).

Este modelo es útil cuando el agua es un factor limitante, sirve para calcular la lámina neta que se debe aplicar con base en el requerimiento hídrico neto del cultivo, en resumen determina la oferta y demanda hídrica del cultivo, según las propiedades físicas del suelo y del manejo agronómico del cultivo, también permite efectuar programaciones de siembras de acuerdo a condiciones del clima y por lo tanto es fundamental para la planeación del riego (Méndez et al., 2013).

Es de gran aplicabilidad para realizar análisis y/o estudios del rendimiento con base en las condiciones hídricas en determinados periodos; por ejemplo, en condiciones de secano o con la lluvia bajo proyecciones ante cambios estimados en el clima (Tavakoli et al., 2015). También es útil para comparar los rendimientos reales de un año determinado en relación con lo que se hubiera debido obtener "en forma teórica". En el mismo sentido sirve como herramienta para diseñar u optimizar sistemas de riego, estimando el au-

6 Una forma de medir el impacto del estrés por déficit de humedad en los cultivos, consiste en estimar la reducción del rendimiento potencial (RRP). Para ello es necesario conocer los valores de RRP bajo un plan de riegos durante el ciclo, que incorporen la información de las variables hidrofísicas del suelo, además de las climatológicas locales (Doorenbos y Pruitt, 1977). 
mento del rendimiento debido al agua adicional y su consecuente variación de rentabilidad en relación a la inversión requerida (Bernal et al., 2012).

Este modelo se ha utilizado principalmente en cultivos de caña de azúcar en el departamento de Valle del Cauca, papa en el altiplano de Cundinamarca y Boyacá, arroz en Tolima y Meta y maíz en la zona productora de Córdoba, Valle del Cauca, Tolima y Meta (Bernal et al., 2013a; Bernal et al., 2013b; Cortés et al., 2013).

Respecto a posibilidades de implementación de los modelos CropWat y AquaCrop, en Colombia se ha propuesto para la alimentación de un modelo a través de escenarios de cambio climático y establecer posibles cambios en la producción y rendimiento de cultivos de arroz de riego, maíz tecnificado y papa (Fernández, 2013). Igualmente para identificar cambios en el rendimiento agrícola en función de la variabilidad climática y de los niveles futuros de $\mathrm{CO}_{2}$ estimados para los cultivos de caña de azúcar en el Valle del Cauca, maíz tecnificado en Córdoba y Meta, papa Diacol Capiro en el altiplano cundiboyacense y maíz amarillo tecnificado en el Tolima y el Valle del Cauca (Bernal et al., 2013a; Bernal et al., 2013b).

Las ventajas de implementar los modelos CropWat y AquaCrop como posibles usos concretos en la agricultura colombiana han sido plateados por Bernal et al. (2013a); Bernal et al. (2013b); Cortés et al. (2013); Méndez et al. (2013) y Bernal et al. (2012):

- "Desarrollar estrategias de irrigación bajo condiciones de déficit hídrico.

- Estudiar el efecto del rendimiento del cultivo en un lugar, el tipo de suelo, la fecha de siembra.

- Analizar el efecto del rendimiento del cultivo de varias técnicas de manejo de tierras.
- Comparar la disponibilidad contra los rendimientos actuales en un campo, granja o una región, identificar las restricciones que limitan la producción del cultivo y la productividad del agua.

- Predecir impacto del cambio climático en la producción de un cultivo.

- Para escenarios de simulación y propósitos de planificación de los economistas, administradores y gerentes del sector hídrico.

- Para facilitar los análisis del rendimiento del cultivo con base en las condiciones hídricas en determinados periodos de tiempo; ya sea en condiciones de secano ó con la lluvia estimada futura".

Para mayor información y consulta de ejemplos típicos de aplicación en el contexto colombiano del programa AquaCrop se puede consultar los siguientes vínculos: www.fao.org/docrep/ field/009/i3427s/i3427s.pdf; www.fao.org/3/ai3429s.pdf

\section{CONCLUSIONES}

La ecuación propuesta por Penman-Monteith que permite calcular la ETo corresponde a un parámetro agroclimático que condensa y resume la influencia de los parámetros meteorológicos en la productividad de las plantas en condiciones de referencia, que luego pueden acercarse a la realidad mediante la utilización de los Kc de cada especie, teniendo en cuenta las características del suelo.

El parámetro Kc resume los requerimientos de agua de la especie de referencia de acuerdo con las condiciones climáticas locales, por lo que la influencia de los eventos ENOS (El Niño y La Niña) sobre la productividad agropecuaria puede calcularse a partir del análisis de las alteraciones sobre los requerimientos de agua de las especies. 
Teniendo en cuenta los cambios que se presentan en la temperatura, humedad del aire, radiación solar y viento, calculadas a través de la ETo y vinculadas con la oferta de lluvias que satisfagan los requerimientos de las especies a través de los balances hídricos agrícolas.

El método de Penman-Monteith y los modelos de simulación agroclimáticos CropWat y AquaCrop son herramientas útiles en los procesos de planeación y de ordenamiento territorial, al facilitar la zonificación agrícola del país, teniendo en cuenta las condiciones climáticas, edáficas y de manejo agronómico de los cultivos.

Estos modelos son una herramienta importante para calcular o predecir el rendimiento esperado de cultivos herbáceos, bajo condiciones climáti- cas normales o de eventos de variabilidad o cambio climático.

Teniendo en cuenta que los modelos de simulación agroclimáticos han sido diseñados para su aplicación en cultivos agroindustriales, se recomienda su evaluación en cultivos de pequeña y mediana escala asociados a la perspectiva de seguridad y soberanía alimentaria.

Finalmente con el fin de ampliar desde el contexto teórico, conceptual y metodológico los modelos de simulación aplicables en Colombia y países similares, se recomiendan como trabajos futuros: aplicación en cultivos promisorios y con ventajas competitivas, diseño de metodologías genéricas adaptables a las condiciones ecosistémicas y sociales a escala territorial.

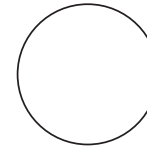

Allen, R., S. Luis, D. Raes y M. Smith. 2006. Evapotranspiración del cultivo. Documento FAO 56. Serie Riego y Drenaje. FAO, Roma.

Andarzian, B., M. Bannayan, P. Steduto, H. Mazraeh, M. E. Barati, M. A. Barati y A. Rahnama. 2011. Validation and testing of the AquaCrop model under full and deficit irrigated wheat production in Iran. Agric. Water Manage. 100(1), 1-8. Doi: 10.1016/j. agwat.2011.08.023

Araya, A., S. Habtu, K. M. Hadgu, A. Kebede y T. Dejene. 2010. Test of AquaCrop model in simulating biomass and yield of water deficient and irrigated barley (Hordeum vulgare). Agric. Water Manage. 97(11), 1838-1846. Doi: 10.1016/j.agwat.2010.06.021

Bernal, J., E. Díaz, J. Méndez. 2012. Uso del modelo AquaCrop para estimar rendimientos agrícolas en Colombia, en el marco del estudio de impactos económicos del cambio climático (EIECC). CP/ COL/3302. IDEAM, Bogotá.

Bernal, J., Díaz, J. Méndez, C. Cortés, F. Boshell, P. Mejías, D. Raes, E. Federes y P. Stedutto. 2013a. Uso del modelo AquaCrop para estimar rendimientos para el cultivo de Caña de Azúcar en el departamento de Valle Del Cauca. FAO Colombia, Bogotá.

\section{REFERENCIAS BIBLIOGRÁFICAS}

Bernal, J., E. Díaz, J. Méndez, C. Cortés, F. Boshell, P. Mejías, D. Raes, E. Federes y P. Stedutto. 2013 b. Uso del modelo AquaCrop para estimar rendimientos para el cultivo de maíz en los departamentos de Córdoba, Meta, Tolima y Valle del Cauca. FAO Colombia, Bogotá.

Boshell, F. 2013. Desarrollo de una función agroclimática para estimar la productividad de los cultivos agrícolas en Colombia. CEPAL-Serie Medio Ambiente y Desarrollo 147, Bogotá.

Bouraima, A., Z. Weihua y W. Chaofu. 2015. Irrigation water requirements of rice using Cropwat model in Northern Benin. Int. J. Agric. Biol. Eng. 8(2), 5864. Doi: 10.3965/j.ijabe.20150802.1290

Bruno, I., A. Silva, K. Reichardt, D. Dourado-Neto, O. Santos y C. Volpe. 2007. Comparison between climatological and field water balances for a coffee crop. Sci. Agric. 64(3), 215-220. Doi: 10.1590/ S0103-90162007000300001

Casa, R., G. Russell y B. Lo Cascio. 2000. Estimation of evapotranspiration from a field of linseed in central Italy. Agric. For. Meteorol. 104(4), 289-301. Doi: 10.1016/S0168-1923(00)00172-6 
Castillo, F., F. Castellví, Á. Bosch, J. Cusidó, J. Sánchez, E. Ferreres, A. Gómez, F. López, J. Martínez, P. Pérez, J. Casanellas, P. Montserrat y J. Villar. 2001. Agrometeorología. Mundi-Prensa, Madrid.

Cavero, J., I. Farre, P. Debaeke y J. Faci. 2000. Simulation of maize yield under stress with the EPICphase and CROPWAT models. Agron. J. 92(4), 679-690. Doi: 10.2134/agronj2000.924679x

Charchousi, D., V.K.. Tsoukala y M.P. Papadopoulou. 2014. How evapotranspiration process may affect the estimation of water footprint indicator in agriculture? Desalination Water Treatment 53(12), 3234-3243. Doi: 10.1080/19443994.2014.934118

Claro, F. 1991. Balance hídrico. HIMAT, Bogotá.

Córdoba-Machado, S., R. Palomino-Lemus, S.R. GámizFortis, Y. Castro-Díez y M.J. Esteban-Parra. 2015. Influence of tropical Pacific SST on seasonal precipitation in Colombia: prediction using El Niño and E1 Niño Modoki. Clim. Dyn. 44(5-6), 1293-1310. Doi: 10.1007/s00382-014-2232-3

Corpoica. 2011. Sistema piloto de alertas agroclimáticas tempranas (SAT), para el apoyo a la toma de decisiones de alternativas tecnológicas de manejo para disminuir los efectos de eventos climáticos adversos en la Sabana de Bogotá, Colombia. En: Taller de Integración de los Sistemas de Alertas Tempranas Hidrometeorológicas. IDEAM, Corporaciones Autónomas Regionales, Bogotá.

Cortés, B., J. Bernal, E. Díaz, J. Méndez, F. Boshell, P. Mejía, D. Raes, E. Federes y P. Stedutto. 2013. Uso del modelo AquaCrop para estimar rendimientos para el cultivo de Arroz en los departamentos de Tolima y Meta. FAO Colombia, Bogotá.

Dastane, N.G. 1978. Effective rainfall in irrigated agricultura. FAO Irrigation and Drainage Paper 25. FAO, Roma.

De Souza, M.J.H., A. Ribeiro, H.G. Leite, F.P. Leite y R.B. Minuzzi. 2006. Disponibilidade hídrica do solo e produtividade do eucalipto em três regiões da Bacia do Rio Doce. Rev. Árvore, 30(3), 399-410. Doi: 10.1590/S0100-67622006000300010

Dechmi, F., E. Playán, J.M. Faci, M. Tejero y A. Bercero. 2003. Analysis of an irrigation district in northeastern Spain II. Irrigation evaluation, simulation and scheduling. Agric. Water Manage. 61(2), 93-109. Doi: 10.1016/S0378-3774(03)00021-0

Doorenbos, J. y W.O. Pruitt. 1977. Guidelines for predicting crop water requirements. Irrigation and Drainage Paper 24. FAO, Roma.

Doorenbos, J., A.H. Kassam, C.I.M. Bentvelsen, V. Branscheid, J.M.G.A. Plusje, M. Smith, G.O. Uit- tenbogaard y H.K. van der Wal. 1979. Efectos del agua sobre el rendimiento de los cultivos. Estudio FAO Riego y Drenaje 33. FAO, Roma.

Echeverría, C., A. Huber y F. Taberlet. 2007. Estudio comparativo de los componentes del balance hídrico en un bosque nativo y una pradera en el sur de Chile. Bosque 28(3), 271-280. Doi: 10.4067/S071792002007000300013

Eslava, J. 1993. Apuntes de climatología y diversidad climática. Rev. Acad. Colomb. Cienc. 18(71), 507-538.

Espinoza, J., J. Ronchail, J. Guyot, G. Cochonneau, F. Naziano, W. Lavado y P. Vauchel. 2009. Spatiotemporal rainfall variability in the Amazon basin countries (Brazil, Perú, Bolivia, Colombia, and Ecuador). Int. J. Climatol. 29(11), 1574-1594. Doi: 10.1002/joc.1791

Fernández, M.E. 2013. Efectos del cambio climático en el rendimiento de tres cultivos mediante el uso del Modelo AquaCrop. FONADE, IDEAM y BID, Bogotá.

FAO. 2006. Crop evapotranspiration: guidelines for computing crop water requirements. Documento 56. FAO, Roma.

FAO. 1992. CROPWAT, a computer program for irrigation planning and management. FAO Irrigation and Drainage Paper 26. FAO, Roma.

FAO. 1977. Las necesidades de agua de los cultivos. Documento 24. FAO, Roma.

Feng, Z., D. Liu y Y. Zhang. 2007. Water requirements and irrigation scheduling of spring maize using GIS and CropWat model in Beijing-Tianjin-Hebei region. Chin. Geogr. Sci. 17(1), 56-63. Doi: 10.1007/ s11769-007-0056-3

Ficklin, D.L., S.L. Letsinger, H. Gholizadeh y J.T. Maxwell. 2015. Incorporation of the PenmanMonteith potential evapotranspiration method into a Palmer Drought Severity Index Tool. Computers Geosciences 85, 136-141. Doi: 10.1016/j.cageo.2015.09.013

García, J., N. Riaño y S. Magnitskiy. 2015. Simulation of corn (Zea mays L.) production in different agricultural zones of Colombia using the AquaCrop model. Agron. Colomb. 32(3), 358-366. Doi: 10.15446/ agron.colomb.v32n3.45939

García-Vila, M. y E. Fereres. 2012. Combining the simulation crop model AquaCrop with an economic model for the optimization of irrigation management at farm level. Europ. J. Agron. 36(1), 21-31. Doi: 10.1016/j.eja.2011.08.003 
Guarnizo, E. 2007. Manejo de aguas. Instituto Colombiano de Desarrollo Rural (Incoder), Agencia de Cooperación Japonesa (JICA). Bogotá.

Jamshid, Y. 2003. The integration of satellite images, GIS and CROPWAT model to investigation of water balance in irrigated area. A case study of Salmas and Tassoj plain, Iran. International Institute for GEO-Information Science and Earth Observation. Enschede, The Netherlands.

Jensen, M., R. Burman y R. Allen. 1990. Evapotranspiration and Irrigation Water Requirements. ASCE Manuals and reports on engineering practices 70. American Society of Civil Engineers, New York, NY.

Jensen, M.E. and H.R. Haise. 1963. Estimating evapotranspiration from solar radiation. J. Irrig. Drainage Div. ASCE 89, 15-41.

Lagos, P., Y. Silva, E. Nickl y K. Mosquera. 2008. El Niño - related precipitation variability in Peru. Adv. Geosci. 14(3), 231-237. Doi: 10.5194/adgeo-14-231-2008

Levine, G., A.D. Cruz-Galvan, D. Garcia, C. Garcés-Restrepo y S. Johnson III. 1998. Performance of two transferred modules in the Lagunera región: water relations. International Water Management Institute. Research Report 23. Colombo, Sri Lanka. En: http://ageconsearch.umn.edu/bitstream/44583/2/ REPORT23.PDF; consulta: mayo de 2015.

Li, F., R. Cao, Y. Zhao, D. Mu, C. Fu y F. Ping. 2015. Remote sensing Penman - Monteith model to estimate catchment evapotranspiration considering the vegetation diversity. Theor. Appl. Climatol. Doi: $10.1007 / \mathrm{s} 00704-015-1628-2$

Liang, L., L. Li, L. Zhang, J. Li y B. Li. 2008. Sensitivity of penman-monteith reference crop evapotranspiration in Tao'er River Basin of northeastern China. Chin. Geogr. Sci. 18(4), 340-347. Doi: 10.1007/ s11769-008-0340-x

López, M., M. Chaves y V. Flórez. 2011. Modelos de cultivos y modelos fenológicos. pp. 153-177. En: Flórez., V.J. (ed.). Sustratos, manejo del clima, automatización y control en sistemas de cultivo sin suelo. Editorial Universidad Nacional de Colombia, Bogotá.

Marcelis, L.F.M, E. Heuvelink y J. Goudriaan. 1998. Modelling biomass production and yield of horticultural crops: a review. Sci. Hortic. 74(1-2), 83111. Doi: 10.1016/S0304-4238(98)00083-1

Méndez, J., B. Cortes, J. Bernal, E. Díaz, F. Boshell, P. Mejía, D. Raes, E. Federes y P. Stedutto. 2013. Uso del modelo AquaCrop para estimar rendimientos para el cultivo de Papa en los departamentos de Cundinamarca y Boyacá. FAO Colombia, Bogotá.
Mullick, M.R.A., M.S. Babel y S.R. Perret. 2011. Discharge-based economic valuation of irrigation water: Evidence from the Teesta River, Bangladesh. Irrig. Drain. 60(4), 481-492. Doi: 10.1002/ird.597

Palacios-Hernández, E., L.E. Carrillo, A. Filonov, L. Brito-Castillo y C. E. Cabrera-Ramos. 2009. Seasonality and anomalies of sea surface temperature off the coast of Nayarit, Mexico. Ocean Dynamics 60(1), 81-91. Doi: 10.1007/s10236-009-0244-z

Penman, H.L. 1948. Natural evaporation from open water, bare soil and grass. Proc. Royal Soc. London. 193, 120-145.

Pereira, A.R., M.B.P. Camargo y N.A. Villa Nova. 2011. Coffee crop coefficient for precision irrigation based on leaf area index. Bragantia 70(4), 946-951. Doi: 10.1590/S0006-87052011000400030

Pérez, A. 1992. Cálculo de la necesidad de agua de las plantas con datos meteorológicos. Ed. Riobamba Pedagógica Freire, Riobamba, Ecuador.

Pérez, J. 2013. Diseño de un sistema de alertas Agroclimáticas tempranas participativas en la subcuenca Río Piedras del Macizo colombiano. Tesis de maestría. Facultad de Ciencias, Universidad Nacional de Colombia, Bogotá.

Priestley, C.H.B. y R.J. Taylor. 1972. On the assessment of surface heat flux and evaporation using largescale parameters. Mon. Weather Rev. 100, 81-82.

Santamaría J, D. Reta, R. Faz y I. Orona. 2008. Reducción del rendimiento potencial en maíz forrajero en calendarios con tres y cuatro riegos. Terra Latinoamericana. 26(3), 235-241, Sociedad Mexicana de la Ciencia del Suelo, A.C. México. En: http:// www.redalyc.org/pdf/573/57313050005.pdf; consulta: mayo de 2015.

Santillán Gutiérrez, E., G. Dávila Vázquez, J. De Anda Sánchez y J.J. Díaz Torres. 2013. Assessment of hydric balance through climatic variables, in the Cazones River Basin, Veracruz, Mexico. Rev. Ambient. Água. 8(3), 104-117. Doi: 10.4136/ambi-agua.1147

Saseendran, S.A., L.R. Ahuja, L. Ma, T.J. Trout, G.S. McMaster, D.C. Nielsen, Q. X. Fang, 2015. Developing and normalizing average corn crop water production functions across years and locations using a system model. Agric. Water Manage. 157, 65-77. Doi: 10.1016/j.agwat.2014.09.002

Silva, V.D.P.R., M.F. Albuquerque, L.E. Araújo, J.H.B.C. Campos, S.L.A. Garcêz y R.S.R. Almeida. 2015. Measurements and modelling of water footprint of sugarcane cultivated in Paraíba State. Rev. Bras. Eng. Agríc. Ambient. 19(6), 521-526. 
Solano, E. y W. Stolz. 2015. El fenómeno ENOS (El niño oscilación del sur), IMN. En: http://www.imn. ac.cr/educacion/enos.html; consulta: junio de 2015.

Spano, D., R.L. Snyder, C. Sirca y P. Duce. 2009. ECOWAT - A model for ecosystem evapotranspiration estimation. Agric. For. Meteorol. 149(10), 1584-1596. Doi: 10.1016/j.agrformet.2009.04.011

Stancalie, G., A. Marica y L. Toulios. 2010. Using earth observation data and CROPWAT model to estimate the actual crop evapotranspiration. Phys. Chem. Earth 35(1-2), 25-30. Doi: 10.1016/j. pce.2010.03.013

Stedutto, P., T. Hsiao, D. Raes y E. Fereres. 2009. Aquacrop - The FAO crop model to simulate yield response to water: I. Concepts and underlying. Agron. J. 101(3), 426-437. Doi: 10.2134/agronj2008.0139s

Tavakoli, A.R., M. Mahdavi Moghadam y A.R. Sepaskhah. 2015. Evaluation of the AquaCrop model for barley production under deficit irrigation and rainfed condition in Iran. Agric. Water Manage. 161, 136-146. Doi: 10.1016/j.agwat.2015.07.020

Toumi, J., S. Er-Raki, J. Ezzahar, S. Khabba, L. Jarlan y A. Chehbouni. 2015. Performance assessment of AquaCrop model for estimating evapotranspiration, soil water content and grain yield of winter wheat in Tensift Al Haouz (Morocco): Application to irrigation management. Agric. Water Manage. 163, 219-235. Doi: 10.1016/j.agwat.2015.09.007

Trenberth, K. 1976. Spatial versus noise in the Southern Oscillation. Mon. Wea. Rev. 112, 323-332.

Turc, L., 1963. Evaluation des besoins en eau d'irrigation, évapotranspiration potentielle, formulation simplifié et mise à jour. Ann. Agron. 12, 13-49.

Vanuytrecht, E., D. Raes, P. Steduto, T.C. Hsiao, E. Fereres, L.K. Heng, M. Garcia, P. Mejias Moreno. 2014. AquaCrop: FAO's crop water productivity and yield response model. Environ. Model. Softw. 62, 351-360. Doi: 10.1016/j.envsoft.2014.08.005

Westerhoff, R.S. 2015. Using uncertainty of Penman and Penman-Monteith methods in combined satellite and ground-based evapotranspiration estimates. Remote Sens. Environ. 169, 102-112. Doi: 10.1016/j.rse.2015.07.021

Wright, J.L. 1982. New evapotranspiration crop coefficients. J. Irrig. Drain. Eng. 108, 57-74.

Yuan, T. y Y. Di. 2014. Variability of lightning flash and thunderstorm over eastern China and Indonesia on ENSO time scales. Atmos. Res. 169, 15-20. Doi: 10.1016/j.atmosres.2015.10.022 